\title{
Solvability of Higher Order (p,q) Laplacian Two-Point Boundary Value Problems
}

\section{Prasad KR ${ }^{1 *}$, Kumar $\mathrm{KR}^{2}$ and Murali $\mathbf{P}^{1}$}

${ }^{1}$ Department of Applied Mathematics, Andhra University, Visakhapatnam, 530 003, India

${ }^{2}$ Department of Mathematics, VITAM College of Engineering, Visakhapatnam, 531 173, India

\begin{abstract}
In this paper, we establish the existence of positive solutions to a coupled system of higher order ( $p, q)$-Laplacian two-point boundary value problem,

$(-1)^{m 1^{-1}}\left[\phi_{p}\left(u^{\left(2 m_{1}\right)}(t)\right)\right]^{\left(m_{1}\right)}=\lambda f_{1}(t, u(t), v(t)), t \in[0,1],(-1)^{n_{2}-1}\left[\phi_{q}\left(u^{\left(m_{2}\right)}(t)\right)\right]^{\left(2 m_{2}\right)}=\lambda f_{2}(t, u(t), v(t)), t \in[0,1], \quad u^{(2 i)}(0)=0=u^{(2 i)}(1), i=0,1,2, \cdots, m_{1}-1$,

$\left[\phi_{p}\left(u^{\left(2 m_{1}\right)}(\mathrm{t})\right)\right]_{a t=0}^{(j)}=0, j=0,1,2, \cdots, n_{1}-2,\left[\phi_{p}\left(u^{\left(2 m_{1}\right)}(\mathrm{t})\right)\right]_{a t t=1}=0,\left[\phi_{q}\left(v^{\left(m_{2}\right)}(\mathrm{t})\right)\right]_{a t=0}^{(2 i)}=0,\left[\phi_{q}\left(v^{\left(m_{2}\right)}(\mathrm{t})\right)\right]_{a t t=1}^{(2 i)}=0, i=0,1,2, \cdots, n_{2}-1$,

$\phi_{p}(y)=y|y|^{p-2}$ by using Guo-Krasnosel'skii fixed point theorem for operators on a cone in a Banach space.
\end{abstract}

Keywords: (p, q)-Laplacian; System of differential equations; Boundary value problem; Green's function; Eigen value; Positive solution; Cone

\section{Introduction}

Differential equations governed by nonlinear differential operators have been widely studied by many researchers. In this theory, the most investigated operator is the classical p-Laplacian, given by $\phi p(y)=y|y| p-2$ with $p>1$. In recent years, it has been generalized to other types of differential operators that preserve the monotonicity of the p-Laplacian, but are not homogeneous. This more general operator is named as $\Phi$-Laplacian, and as involved in the modeling of non-Newtonian fluid theory, diffusion of flows in porous media, nonlinear elasticity and theory of capillary surfaces. Much interest has been created in establishing positive solutions and multiple positive solutions for two-point, multi-point boundary value problems (BVPs) associated with ordinary differential equations. To mention the related papers along these lines, we refer to Erbe et al., Eloe et al., Wong et al., Davis et al., Henderson et al., Ntouyas et al. and Li [1-7]. Due to wide mathematical and physical background the existence of positive solutions for nonlinear BVPs with p-Laplacian operator have received wide attention. To mention a few, Agarwal, Bohner, Agarwal, Avery and $\mathrm{Chu}$ [8-12], and the references therein.

Recently, Prasad et al. [13] established positive solutions for ( $p$, q)-Laplacian boundary value problem. In this paper, we consider a coupled system of higher order ( $p, q)$-Laplacian two-point boundary value problem,

$$
\left.\begin{array}{l}
(-1)^{m 1^{-1}}\left[\phi_{p}\left(u^{\left(2 m_{1}\right)}(t)\right)\right]^{\left(n_{1}\right)}=\lambda f_{1}(t, u(t), v(t)), t \in[0,1], \\
(-1)^{n_{2}-1}\left[\phi_{q}\left(u^{\left(m_{2}\right)}(t)\right)\right]^{\left(2 n_{2}\right)}=\lambda f_{2}(t, u(t), v(t)), t \in[0,1], \\
u^{(2 i)}(0)=0=u^{(2 i)}(1), i=0,1,2, \cdots, \mathrm{m}_{1}-1, \\
{\left[\phi_{p}\left(u^{\left(2 m_{1}\right)}(t)\right)\right]_{a t t=0}^{(j)}=0, j=0,1,2, \cdots, n_{1}-2,} \\
{\left[\phi_{p}\left(u^{\left(2 m_{1}\right)}(t)\right)\right]_{a t t=1}=0,}
\end{array}\right\}
$$

Where

$m_{1}, n_{1}, m_{2}, n_{2} € N, n_{1} \geq 2, m_{2} \geq 2$ and $\frac{1}{p}+\frac{1}{q}=1$

We assume the following conditions hold throughout this paper:

(A1) $\mathrm{f}_{\mathrm{i}}:[0,1] \times \mathrm{R}^{2} \rightarrow[0, \infty)$ are continuous functions, for $\mathrm{i}=1,2$, (A2) each of

$$
\begin{aligned}
& f_{1,0}=\lim _{u+v \rightarrow 0^{+}} \frac{f_{1}(t, u(t), v(t))}{\phi_{p}(u+v)}, f_{2,0}=\lim _{u+v \rightarrow 0^{+}} \frac{f_{2}(t, u(t), v(t))}{\phi_{q}(u+v)}, \\
& f_{1, \infty}=\lim _{u+v \rightarrow \infty} \frac{f_{1}(t, u(t), v(t))}{\phi_{p}(u+v)}, f_{2, \infty}=\lim _{u+v \rightarrow \infty} \frac{f_{2}(t, u(t), v(t))}{\phi_{q}(u+v)},
\end{aligned}
$$

exist as positive real numbers.

To obtain a solution of the BVP (1.1)-(1.4), we construct the Green functions for the corresponding homogeneous BVPs. For $n_{1} \geq 2$ let $\mathrm{G}(\mathrm{t}, \mathrm{s})$ be the Green's function of the BVP

$$
\begin{aligned}
& -x^{\left(n_{1}\right)}(t)=0, t \in[0,1], \\
& x^{(j)}(0)=0, j=0,1,2, \cdots, n_{1}-2, x(1)=0,
\end{aligned}
$$

${ }^{*}$ Corresponding author: Prasad KR, Department of Applied Mathematics, Andhra University, Visakhapatnam, 530 003, India, Tel: 891275 4586; E-mail: rajendra92@rediffmail.com

Received July 21, 2014; Accepted August 19, 2014; Published August 25, 2014

Citation: Prasad KR, Kumar KR, Murali P (2014) Solvability of Higher Order ( $p, q)$ Laplacian Two-Point Boundary Value Problems. J Appl Computat Math 3: 182 doi:10.4172/2168-9679.1000182

Copyright: ( 2014 Prasad KR, et al. This is an open-access article distributed under the terms of the Creative Commons Attribution License, which permits unrestricted use, distribution, and reproduction in any medium, provided the original author and source are credited. 
and after simple computation it can be obtained as

$$
G(t, s)= \begin{cases}\frac{t^{n_{1}-1}(1-s)^{n_{1}-1}}{\left(n_{1}-1\right) !}, & 0 \leq t \leq s \leq 1, \\ \frac{t^{n_{1}-1}(1-s)^{n_{1}-1}}{\left(n_{1}-1\right) !}-\frac{(t-s)^{n_{1}-1}}{\left(n_{1}-1\right) !}, & 0 \leq s \leq t \leq 1 .\end{cases}
$$

Let $\mathrm{H}_{\mathrm{m} 1}(\mathrm{t}, \mathrm{s})$ be the Green's function of the homogeneous BVP,

$$
\begin{aligned}
& (-1)^{m_{1}} y^{\left(2 m_{1}\right)}(t)=0, t \in[0,1], \\
& y^{(2 i)}(0)=0=y^{(2 i)}(1), i=0,1, \cdots, m_{1}-1,
\end{aligned}
$$

and is recursively defined as,

$$
H_{m 1}(t, s)=\int_{0}^{1} H_{m 1-1}(t, r) H_{1}(r, s) d r,
$$

where $\mathrm{H}_{1}(\mathrm{t}, \mathrm{s})$ is the Green's function of the BVP,

$-y^{\prime \prime}(t)=0, y(0)=0=y(1)$, and is given by

$$
H_{1}(t, s)= \begin{cases}t(1-s), & 0 \leq t \leq s<1, \\ s(1-t), & 0<s \leq t \leq 1 .\end{cases}
$$

Then, the equivalent integral equation for (1.1) and (1.3) is

$$
\begin{gathered}
u(t)=\lambda \int_{0}^{1} H_{m 1}(t, s) \phi_{p}^{-1}\left(\int_{0}^{1} G(s, \tau) f_{1}(\tau, u(\tau), v(\tau)) d \tau\right) d s \\
\lambda \int_{0}^{1} H_{m 1}(t, s) \phi_{q}\left(\int_{0}^{1} G(s, \tau) f_{1}(\tau, u(\tau), v(\tau)) d \tau\right) d s .
\end{gathered}
$$

Similarly for $\mathrm{m}_{2} \geq 2$, let $\mathrm{H}(\mathrm{t}, \mathrm{s})$ be the Green's function of the BVP,

$$
-x^{\left(m_{2}\right)}(t)=0, t \in[0,1]
$$$$
x^{(j)}(0)=0, j=0,1,2, \cdots, m_{2}-2, x(1)=0,
$$

and after simple computation it can be obtained as

$$
H(t, s)= \begin{cases}\frac{t^{m_{2}-1}(1-s)^{m_{2}-1}}{\left(m_{2}-1\right) !}, & 0 \leq t \leq s<1, \\ \frac{t^{m_{2}-1}(1-s)^{m_{2}-1}}{\left(m_{2}-1\right) !}-\frac{(t-s)^{m_{2}-1}}{\left(m_{2}-1\right) !}, & 0<s \leq t \leq 1 .\end{cases}
$$

Let $\mathrm{G}_{\mathrm{n} 2}(\mathrm{t}, \mathrm{s})$ be the Green's function of the homogeneous BVP,

$$
(-1)^{n_{2}} y^{\left(2 n_{2}\right)}(t)=0, t \in[0,1]
$$$$
y^{(2 i)}(0)=0=y^{(2 i)}(1), \mathrm{i}=0,1, \cdots, n_{2}-1,
$$

and it can be recursively defined as

$$
G_{n 2}(t, s)=\int_{0}^{1} G_{n 2-1}(t, r) G_{1}(r, s) d r,
$$

where $G_{1}(t, s)$ is the Green's function of the BVP,

$$
-y^{\prime \prime}(\mathrm{t})=0, \mathrm{y}(0)=0=\mathrm{y}(1),
$$

and is given by

$$
G_{1}(t, s)=H_{1}(t, s) \text {. }
$$

Then, the equivalent integral equation for (1.2) and (1.4) is

$$
v(t)=\lambda \int_{0}^{1} H(t, s) \phi_{q}^{-1}\left(\int_{0}^{1} G_{n 2}(s, \tau) f_{2}(\tau, u(\tau), v(\tau)) d \tau\right) d s
$$

$$
=\lambda \int_{0}^{1} H(t, s) \phi_{p}\left(\int_{0}^{1} G_{n 2}(s, \tau) f_{2}(\tau, u(\tau), v(\tau)) d \tau\right) d s
$$

Further, it is easily seen that $\mathrm{H}_{\mathrm{m} 1}(\mathrm{t}, \mathrm{s}), \mathrm{H}(\mathrm{t}, \mathrm{s}), \mathrm{G}_{\mathrm{n} 2}(\mathrm{t}, \mathrm{s})$ and $\mathrm{G}(\mathrm{t}, \mathrm{s})$ are all non-negative on $[0,1] \times[0,1]$.

AsolutionoftheBVP(1.1)-(1.4), isafunction $(u, v) \in c^{2 m_{1}}[0,1] \times c^{m_{2}}[0,1]$ such that $\left(\phi_{p} \circ u^{\left(2 m_{1}\right)}, \phi_{q} \circ v^{\left(m_{2}\right)}\right) \in c^{n_{1}}[0,1] \times c^{2 n_{2}}[0,1]$ and $(\mathrm{u}, \mathrm{v})$ satisfies the $\operatorname{BVP}(1.1)-(1.4)$.

A positive solution $(\mathrm{u}, \mathrm{v})$ of the BVP (1.1)-(1.4) is a solution of the BVP (1.1)-(1.4) such that $u$ and $v$ are non-negative on $[0,1]$. The rest of the paper is organized as follows. In Part 4 , we estimate the bounds of the Green functions which will be used in defining the positive operator. In Part 5, we provide an appropriate Banach space and a cone in order to apply the Guo-Krasnosel'skii fixed point theorem [14] to characterize the values of $\lambda$ so that the BVP (1.1)-(1.4) has a positive solution. Finally as an application, we give an example to demonstrate our result

\section{Bounds of the Green's Functions}

In this section, we state some lemmas to estimate the bounds of the Green functions which are needed later. Let $I=\left[\frac{1}{4}, \frac{3}{4}\right]$. For the
preceding Lemmas we refer the reader to [13].

Lemma 2.1 [13] For $(t, s) \in I \times[0,1]$,

$G_{j}(t, s) \geq\left(\frac{11}{6}\right)^{j-1} \frac{1}{4^{3 j-2}}(1-s) s$

Lemma 2.2 For $\mathrm{t}, \mathrm{s} \in[0,1]$,

$G_{j}(t, s) \geq \frac{1}{6^{j-1}}(1-s) s$

Lemma 2.3 For $(\mathrm{t}, \mathrm{s}) \in \mathrm{I} \times[0,1]$,

$H_{j}(t, s) \geq\left(\frac{11}{6}\right)^{j-1} \frac{1}{4^{3 j-2}}(1-s) s$

Lemma 2.4 For $t, s \in[0,1]$,

$H_{j}(t, s) \leq \frac{1}{6^{j-1}}(1-s) s$

Lemma 2.5 For $(t, s) \in \mathrm{I} \times[0,1]$,

$H(t, s) \geq \frac{1}{4^{m_{2}-1}} \mathrm{H}(\mathrm{s}, \mathrm{s})$.

Lemma 2.6 For $t, s \in[0,1]$,

$H(t, s) \leq \mathrm{H}(\mathrm{s}, \mathrm{s})$.

Lemma 2.7 For $(\mathrm{t}, \mathrm{s}) \in \mathrm{I} \times[0,1]$,

$G(t, s) \geq \frac{1}{4^{n_{1}-1}} G(s, s)$.

Lemma 2.8 For $t, s \in[0,1]$,

$G(t, s) \leq G(s, s)$.

Denote

$$
M=\min \left\{\frac{11^{m_{1}-1}}{4^{3 m_{1}-2}} \cdot \phi_{p}^{-1}\left(\frac{1}{4^{n_{1}-1}}\right), \frac{1}{4^{m_{2}-1}} \cdot \phi_{q}^{-1}\left(\frac{11^{n_{2}-1}}{4^{3 n_{2}-2}}\right)\right\} .
$$

The values of the parameter $\lambda$ will be determined for which there exist positive solutions of the BVP (1.1)-(1.4), using the following GuoKrasnosel'skii fixed point theorem [14]. 
Theorem 2.9 [14] Let X be a Banach Space, $\kappa \subseteq X$ be a cone and suppose that $\Omega_{1}, \Omega_{2}$ are open subsets of X with $0 \in \Omega_{1}$ and $\bar{\Omega}_{1} \subset \Omega_{2}$. Suppose further that $T: k \cap\left(\bar{\Omega}_{2} \backslash \Omega_{1}\right) \rightarrow k$ is completely continuous operator such that either

i) $\|T u\| \leq\|u\|, u \in k \cap \partial \Omega_{1}$ and $\|T u\| \geq\|u\|, u \in k \cap \partial \Omega_{2}$ or

ii) $\|T u\| \geq\|u\|, u \in k \cap \partial \Omega_{1}$ and $\|T u\| \leq\|u\|, u \in k \cap \partial \Omega_{2}$ holds

Then $\mathrm{T}$ has a fixed point in $k \cap\left(\bar{\Omega}_{2} \backslash \Omega_{1}\right)$.

\section{Positive Solution in a Cone}

In this section, we establish the criteria to determine eigen values for which the BVP (1.1)-(1.4) has at least one positive solution. Consider the Banach space $\mathrm{E}=\{\mathrm{y}: \mathrm{y} \in \mathrm{C}[0,1]\}$ with the norm $/ / \bullet / /$ and the Banach space $B=E \times E$, with the norm $/ /(\mathrm{u}, \mathrm{v}) / /=/ / \mathrm{u} / /_{0}+/ / \mathrm{v} / /_{0}$, where $\|u\|_{0}=\max _{t \in[0,1]}|u(t)|$.

Define the cone $\mathrm{P} \subset \mathrm{B}$ by,

$$
\begin{aligned}
& P=\{(u, v) \in B \mid u(t) \geq 0, v(t) \geq 0 \text { on }[0,1] \text { and } \\
& \left.\qquad \min _{t \in I}\{u(t)+v(t)\} \geq M\|(u, v)\|\right\} .
\end{aligned}
$$

For our first result, we define the positive numbers $\mathrm{R}_{1}, \mathrm{R}_{2}$ by

$$
\begin{aligned}
& R_{1}=\max \left\{\left[\frac{2 M^{2}}{6^{m 1}} \phi_{p}^{-1}\left(\int_{0}^{1} G(\tau, \tau) f_{1, \infty} d \tau\right)\right]^{-1},\right. \\
& {\left.\left[2 M^{2} \int_{0}^{1} H(s, s) d s \phi_{q}^{-1}\left(\frac{1}{6^{n_{2}}} f_{2, \infty}\right)\right]^{-1}\right\} }
\end{aligned}
$$

And

$$
\begin{aligned}
& R_{2}=\min \left\{\left[\frac{2}{6^{m 1}} \phi_{p}^{-1}\left(\int_{0}^{1} G(\tau, \tau) f_{1,0} d \tau\right)\right]^{-1},\right. \\
& {\left.\left[2 \int_{0}^{1} H(s, s) d s \phi_{q}^{-1}\left(\frac{1}{6^{n_{2}}} f_{2,0}\right)\right]^{-1}\right\} . }
\end{aligned}
$$

Theorem 3.1 Assume that the conditions $\left(\mathrm{A}_{1}\right),\left(\mathrm{A}_{2}\right)$ are satisfied. Then, for each $\lambda$ satisfying

$$
R_{1}<\lambda<R_{2}
$$

There exists at least one positive solution (u, v) of the BVP (1.1)-(1.4).

Proof: Let $\lambda$ be as in (3.1). And let $\epsilon>0$ be chosen such that

$$
\begin{aligned}
& \max \left\{\left[\frac{2 M^{2}}{6^{m_{1}}} \phi_{p}^{-1}\left(\int_{0}^{1} G(\tau, \tau)\left(f_{1, \infty}-\epsilon\right) d \tau\right)\right]^{-1},\right. \\
& {\left.\left[2 M^{2} \int_{0}^{1} H(s, s) d s \phi_{q}^{-1}\left(\frac{1}{6^{n_{2}}}\left(f_{2, \infty}-\epsilon\right)\right)\right]^{-1}\right\} \leq \lambda }
\end{aligned}
$$

And

$$
\begin{aligned}
& \lambda \leq \min \left\{\left[\frac{2}{6^{m_{1}}} \phi_{p}^{-1}\left(\int_{0}^{1} G(\tau, \tau)\left(f_{1,0}+\in\right) d \tau\right)\right]^{-1},\right. \\
& {\left.\left[2 \int_{0}^{1} H(s, s) d s \phi_{q}^{-1}\left(\frac{1}{6^{n_{2}}}\left(f_{2,0}+\in\right)\right)\right]^{-1}\right\} . }
\end{aligned}
$$

The integral operator $\mathrm{T}: \mathrm{P} \rightarrow \mathrm{B}$ is defined by

$$
\begin{aligned}
T(u, v)=\left(\lambda \int_{0}^{1} H_{m_{1}}(t, s) \phi_{p}^{-1}\left(\int_{0}^{1} G(s, \tau) f_{1}(\tau, u(\tau), v(\tau)) d \tau\right) d s,\right. \\
\left.\quad \lambda \int_{0}^{1} H(t, s) \phi_{q}^{-1}\left(\int_{0}^{1} G_{n_{2}}(s, \tau) f_{2}(\tau, u(\tau), v(\tau)) d \tau\right) d s\right) .
\end{aligned}
$$

And also we denote

$$
\begin{aligned}
& T_{1}(u, v)=\lambda \int_{0}^{1} H_{m_{1}}(t, s) \phi_{p}^{-1}\left(\int_{0}^{1} G(s, \tau) f_{1}(\tau, u(\tau), v(\tau)) d \tau\right) d s, \\
& \left.T_{2}(u, v)=\lambda \int_{0}^{1} H(t, s) \phi_{q}^{-1}\left(\int_{0}^{1} G_{n_{2}}(s, \tau) f_{2}(\tau, u(\tau), v(\tau)) d \tau\right) d s\right) .
\end{aligned}
$$

From (A1) and the positivity of the Green functions that, for $(\mathrm{u}, \mathrm{v})$ $\in \mathrm{P}, \mathrm{T} 1(\mathrm{u}, \mathrm{v})(\mathrm{t}) \geq 0, \mathrm{~T} 2(\mathrm{u}, \mathrm{v})(\mathrm{t}) \geq 0$, for $\mathrm{t} \in[0,1]$. Now for $(\mathrm{u}, \mathrm{v}) \in \mathrm{P}$ and Lemma 2.4, Lemma 2.8, we have

$$
\begin{aligned}
T_{1}(u, v)(\mathrm{t}) & =\lambda \int_{0}^{1} H_{m_{1}}(t, s) \phi_{p}^{-1}\left(\int_{0}^{1} G(s, \tau) f_{1}(\tau, u(\tau), v(\tau)) d \tau\right) d s \\
& \leq \lambda \frac{1}{6^{m 1-1}} \int_{0}^{1}(1-s) s \phi_{p}^{-1}\left(\int_{0}^{1} G(\tau, \tau) f_{1}(\tau, u(\tau), v(\tau)) d \tau\right) d s \\
& \leq \lambda \frac{1}{6^{m_{1}}} \phi_{p}^{-1}\left(\int_{0}^{1} G(\tau, \tau) f_{1}(\tau, u(\tau), v(\tau)) d \tau\right)
\end{aligned}
$$

so that

$$
\left\|T_{1}(u, v)\right\| 0 \leq \lambda \frac{1}{6^{m_{1}}} \phi_{p}^{-1}\left(\int_{0}^{1} G(\tau, \tau) f_{1}(\tau, u(\tau), v(\tau)) d \tau\right) .
$$

Now for $(\mathrm{u}, \mathrm{v}) \in \mathrm{P}$ from (2.9), Lemma 2.3 and Lemma 2.7, we have

$$
\begin{aligned}
& \min _{t \in I} T_{1}(u, v)(t)= \min _{t \in I} \lambda \int_{0}^{1} H_{m_{1}}(t, s) \phi_{p}^{-1}\left(\int_{0}^{1} G(s, \tau) f_{1}(\tau, u(\tau), v(\tau)) d \tau\right) d s \\
& \geq \lambda\left(\frac{11}{6}\right)^{m_{1}-1} \frac{1}{4^{3 m_{1}-2}} \int_{0}^{1}(1-s) S \\
& \quad \times \phi_{p}^{-1}\left(\frac{1}{4^{n_{1}-1}} \int_{0}^{1} G(\tau, \tau) f_{1}(\tau, u(\tau), v(\tau)) d \tau\right) d s \\
& \geq \lambda \frac{11^{m_{1}-1}}{4^{3 m_{1}-2}} \frac{1}{6^{m_{1}-1}} \int_{0}^{1}(1-\mathrm{s}) \mathrm{s} \phi_{p}^{-1}\left(\frac{1}{4^{n_{1}-1}}\right) \\
& \times \phi_{p}^{-1}\left(\int_{0}^{1} G(\tau, \tau) f_{1}(\tau, u(\tau), v(\tau)) d \tau\right) d s
\end{aligned}
$$

$$
\begin{aligned}
& \geq \lambda \frac{11^{m_{1}-1}}{4^{3 m_{1}-2}} \cdot \phi_{p}^{-1}\left(\frac{1}{4^{n_{1}-1}}\right) \\
& \quad \times \frac{1}{6^{m_{1}}} \phi_{p}^{-1}\left(\int_{0}^{1} G(\tau, \tau) f_{1}(\tau, u(\tau), v(\tau)) d \tau\right) \\
& \geq M\left\|T_{1}(u, v)\right\|_{0} .
\end{aligned}
$$

In a similar manner, we conclude that

$\min _{t \in I} T_{2}(u, v)(t) \geq M\left\|T_{2}(u, v)\right\|_{0}$.

Therefore,

$$
\begin{aligned}
\min _{t \in I}\left\{T_{1}(u, v)(t)+T_{2}(u, v)(t)\right\} & \geq M\left\|T_{1}(u, v)\right\|_{0}+M\left\|T_{2}(u, v)\right\|_{0} \\
& =M\|T(u, v)\| .
\end{aligned}
$$

Thus, $\mathrm{T}: \mathrm{P} \rightarrow \mathrm{P}$. Further, the operator $\mathrm{T}$ is completely continuous by an application of the Arzela-Ascoli theorem.

Now, from the definitions of $f_{1,0}$ and $f_{2,0}$, there exists an $K_{1}>0$ such 
$f_{1}(t, u(t), v(t)) \leq\left(f_{1,0}+\in\right) \phi_{p}(u+v), 0<\phi_{p}(u+v) \leq K_{1}$,

$f_{2}(t, u(t), v(t)) \leq\left(f_{2,0}+\in\right) \phi_{q}(u+v), 0<\phi_{q}(u+v) \leq K_{1}$.

Let $(\mathrm{u}, \mathrm{v}) \in \mathrm{P}$ with $\|(\mathrm{u}, \mathrm{v})\|=\mathrm{K} 1$. Then, from Lemma 2.4 , Lemma 2.8 and choice of $\epsilon$,

$$
\begin{aligned}
T_{1}(u, v)(\mathrm{t}) & =\lambda \int_{0}^{1} H_{m_{1}}(t, s) \phi_{p}^{-1}\left(\int_{0}^{1} G(s, \tau) f_{1}(\tau, u(\tau), v(\tau)) d \tau\right) d s \\
& \leq \lambda \frac{1}{6^{m-1}} \int_{0}^{1}(1-s) s \phi_{p}^{-1}\left(\int_{0}^{1} G(\tau, \tau) f_{1}(\tau, u(\tau), v(\tau)) d \tau\right) d s \\
& \leq \lambda \frac{1}{6^{m_{1}}} \phi_{p}^{-1}\left(\int_{0}^{1} G(\tau, \tau)\left(f_{1,0}+\epsilon\right) d \tau\right)(u+v) \\
& \leq \lambda \frac{1}{6^{m_{1}}} \phi_{p}^{-1}\left(\int_{0}^{1} G(\tau, \tau)\left(f_{1,0}+\in\right) d \tau\right)\|(u, v)\| \\
& \leq \frac{1}{2}\|(u, v)\|=\frac{K_{1}}{2} .
\end{aligned}
$$

In a similar manner, we conclude that

$$
\begin{aligned}
T_{2}(u, v)(\mathrm{t}) & =\lambda \int_{0}^{1} H(t, s) \phi_{q}^{-1}\left(\int_{0}^{1} G_{n_{2}}(s, \tau) f_{2}(\tau, u(\tau), v(\tau)) d \tau\right) d s \\
& \leq \frac{1}{2}\|(u, v)\|=\frac{K_{1}}{2} .
\end{aligned}
$$

Hence, $\|\mathrm{T}(\mathrm{u}, \mathrm{v})\| \leq \mathrm{K} 1=\|(\mathrm{u}, \mathrm{v})\|$. If we set

$$
\Omega_{1}=\left\{(\mathrm{u}, \mathrm{v}) \in \mathrm{B} \mid\|(\mathrm{u}, \mathrm{v})\|<\mathrm{K}_{1}\right\}
$$

Then

$$
\|T(u, v)\| \leq\|(u, v)\|,(u, v) \in P \cap \partial \Omega_{1} .
$$

Next, from the definitions of $\mathrm{f}_{1, \infty}$ and $\mathrm{f}_{2, \infty}$, there exists $\bar{K}_{2}>0$ such that

$$
f_{1}(t, u(t), v(t)) \geq\left(f_{1, \infty}-\epsilon\right) \phi_{p}(u+v), \quad \phi_{p}(u+v) \geq \bar{K}_{2},
$$$$
f_{2}(t, u(t), v(t)) \geq\left(f_{2, \infty}-\in\right) \phi_{q}(u+v), \quad \phi_{q}(u+v) \geq \bar{K}_{2} \text {. }
$$$$
\text { Let } K_{2}=\max \left\{2 K_{1}, \frac{\bar{K}_{2}}{M}\right\} \text {. }
$$

Choose $(\mathrm{u}, \mathrm{v}) \in \mathrm{P}$ with $\|(\mathrm{u}, \mathrm{v})\|=\mathrm{K}_{2}$. Then,

$$
\min _{t \in I}\{u(t)+v(t)\} \geq M\|(u, v)\| \geq \bar{K}_{2} .
$$

Consequently, from (2.9), Lemma 2.3, Lemma 2.7 and choice $\epsilon$,

$$
\begin{aligned}
T_{1}(u, v)(\mathrm{t})= & \lambda \int_{0}^{1} H_{m_{1}}(t, s) \phi_{p}^{-1}\left(\int_{0}^{1} G(s, \tau) f_{1}(\tau, u(\tau), v(\tau)) d \tau\right) d s \\
\geq & \lambda\left(\frac{11}{6}\right)^{m_{1}-1} \frac{1}{4^{3 m_{1}-2}} \int_{0}^{1}(1-s) \mathrm{s} \\
& \times \phi_{p}^{-1}\left(\frac{1}{4^{n_{1}-1}} \int_{0}^{1} G(\tau, \tau) f_{1}(\tau, u(\tau), v(\tau)) d \tau\right) d s \\
\geq & \lambda \frac{11^{m_{1}-1}}{4^{3 m_{1}-2}} \frac{1}{6^{m_{1}-1}} \int_{0}^{1}(1-s) s \phi_{p}^{-1}\left(\frac{1}{4^{n_{1}-1}}\right) \\
& \geq \lambda \frac{1}{6^{m_{1}}} \frac{11^{m_{1}-1}}{4^{3 m_{1}-2}} \cdot \phi_{p}^{-1}\left(\frac{1}{4^{n_{1}-1}}\right) \\
& \times \lambda \frac{1}{6^{m_{1}}} M \phi_{p}^{-1}\left(\int_{0}^{1} G(\tau, \tau)\left(\int_{0}^{1} G(\tau, \tau)\left(f_{1, \infty}-\in\right) d \tau\right)(u+v)\right. \\
& \geq \lambda \frac{M^{2}}{6^{m_{1}}} \phi_{p}^{-1}\left(\int_{0}^{1} G(\tau, \tau)\left(f_{1, \infty}-\in\right) d \tau\right)\|(u, v)\| \\
& \geq \frac{1}{2}\|(u+v)\|=\frac{K_{2}}{2} .
\end{aligned}
$$

In a similar manner, we conclude that

$$
\begin{aligned}
T_{2}(u, v)(\mathrm{t}) & =\lambda \int_{0}^{1} H(t, s) \phi_{q}^{-1}\left(\int_{0}^{1} G_{n_{2}}(s, \tau) f_{2}(\tau, u(\tau), v(\tau)) d \tau\right) d s \\
& \geq \frac{1}{2}\|(u+v)\|=\frac{K_{2}}{2} .
\end{aligned}
$$

Therefore, $T_{2}(u, v)(\mathrm{t}) \geq K_{2}$.

Hence, $\|T(u, v)\| \geq K_{2}=\|(u, v)\|$. If we set,

$\Omega_{2}=\left\{(u, v) \in B \mid\|(u, v)\|<K_{2}\right\}$,

Then $\|T(u, v)\| \geq\|(u, v)\|$ for $(u, v) \in P \cap \partial \Omega_{2}$

Applying Theorem 2.9 to (3.3) and (3.4) we obtain that $\mathrm{T}$ has a fixed point (u,v) in $P \cap\left(\bar{\Omega}_{2} \backslash \Omega_{1}\right)$ and hence the BVP (1.1)-(1.4) has a positive solution such that $\mathrm{K}_{1} \leq\|(\mathrm{u}, \mathrm{v})\| \leq \mathrm{K}_{2}$. The proof is complete.

For our next result, we define the positive numbers $\mathrm{R}_{3}, \mathrm{R}_{4}$ by

$$
\begin{aligned}
& R_{3}=\max \left\{\left[\frac{2 M^{2}}{6^{m_{1}}} \phi_{p}^{-1}\left(\int_{0}^{1} G(\tau, \tau) d \tau f_{1,0}\right)\right]^{-1},\right. \\
& {\left.\left[2 M^{2} \int_{0}^{1} H(s, s) \phi_{p}^{-1}\left(\frac{1}{6^{n_{2}}} f_{2,0}\right) d s\right]^{-1}\right\} }
\end{aligned}
$$

and

$$
\begin{aligned}
& R_{4}=\min \left\{\left[\frac{2}{6^{m_{1}}} \phi_{p}^{-1}\left(\int_{0}^{1} G(\tau, \tau) d \tau f_{1, \infty}\right)\right]^{-1},\right. \\
& {\left.\left[2 \int_{0}^{1} H(s, s) \phi_{q}^{-1}\left(\frac{1}{6^{n_{2}}} f_{2, \infty}\right) d s\right]^{-1}\right\} . }
\end{aligned}
$$

Theorem 3.2 Assume that the conditions (A1), (A2) are satisfied. Then, for each $\lambda$ satisfying

$$
R_{3}<<R_{4}
$$

there exists at least one positive solution $(\mathrm{u}, \mathrm{v})$ of the BVP (1.1)-(1.4).

Proof: Let $\lambda$ be as in (3.5). And let $\epsilon>0$ be chosen such that

$$
\begin{aligned}
& m \operatorname{ax}\left\{\left[\frac{2 M^{2}}{6^{m_{1}}} \phi_{p}^{-1}\left(\int_{0}^{1} G(\tau, \tau)\left(f_{1,0}-\epsilon\right) d \tau\right)\right]^{-1},\right. \\
& {\left.\left[2 M^{2} \int_{0}^{1} H(s, s) \mathrm{ds} \phi_{q}^{-1}\left(\frac{1}{6^{n_{2}}}\left(f_{2,0}-\epsilon\right)\right)\right]^{-1}\right\} \leq \lambda }
\end{aligned}
$$

and

$$
\begin{aligned}
& \lambda \leq \min \left\{\left[\frac{2}{6^{m_{1}}} \phi_{q}^{-1}\left(\int_{0}^{1} G(\tau, \tau)\left(f_{1, \infty}+\in\right) d \tau\right)\right]^{-1},\right. \\
& {\left.\left[2 \int_{0}^{1} H(s, s) d s \phi_{p}^{-1}\left(\frac{1}{6^{n_{2}}}\left(f_{2, \infty}+\epsilon\right)\right)\right]^{-1}\right\} . }
\end{aligned}
$$

Let $\mathrm{T}$ be the cone preserving completely continuous operator that was defined by (3.2). From the definitions of $f_{1,0}$ and $f_{2,0}$, there exists $\mathrm{J}_{1}>0$ such that

$$
\begin{array}{ll}
f_{1}(t, u(t), v(t)) \geq\left(f_{1,0}-\in\right) \phi_{p}(u+v), & \phi_{p}(u+v) \leq J_{1}, \\
f_{2}(t, u(t), v(t)) \geq\left(f_{2,0}-\in\right) \phi_{q}(u+v), & \phi_{q}(u+v) \leq J_{1} .
\end{array}
$$

Choose (u, v) $\in \mathrm{P}$ with $\|(\mathrm{u}, \mathrm{v})\|=\mathrm{J}_{1}$. Then, from (2.9), Lemma 2.3 and Lemma 2.7 we have 


$$
\begin{aligned}
T_{1}(u, v)(\mathrm{t})= & \lambda \int_{0}^{1} H_{m_{1}}(t, s) \phi_{p}^{-1}\left(\int_{0}^{1} G(s, \tau) f_{1}(\tau, u(\tau), v(\tau)) d \tau\right) d s \\
& \geq \lambda\left(\frac{11}{6}\right)^{m_{1}-1} \frac{1}{4^{3 m_{1}-2}} \int_{0}^{1}(1-s) \mathrm{s} \\
& \times \phi_{p}^{-1}\left(\frac{1}{4^{n_{1}-1}} \int_{0}^{1} G(\tau, \tau) f_{1}(\tau, u(\tau), v(\tau)) d \tau\right) d s \\
\geq & \lambda \frac{11^{m_{1}-1}}{4^{3 m_{1}-2}} \frac{1}{6^{m_{1}-1}} \int_{0}^{1}(1-s) s \phi_{p}^{-1}\left(\frac{1}{4^{n_{1}-1}}\right) \\
\geq & \lambda \frac{1}{6^{m_{1}}} \frac{11^{m_{1}-1}}{4^{3 m_{1}-2}} \cdot \phi_{p}^{-1}\left(\frac{1}{4^{n_{1}-1}}\right) \\
& \times \lambda \frac{M}{6^{m_{1}}} \phi_{p}^{-1}\left(\int_{0}^{1} G(\tau, \tau)\left(\int_{0}^{1} G(\tau, \tau)\left(f_{1,0}-\epsilon\right) \phi_{p}(u+v) d \tau\right)\right. \\
\geq & \left.\left.\lambda \frac{M^{2}}{6^{m_{1}}} \phi_{p}^{-1}\left(\int_{0}^{1} G(\tau, \tau)\left(f_{1,0}-\in\right) d \tau\right)(u+v) d \tau\right) d s\right)\|(u, v)\| \\
\geq & \frac{1}{2}\|(u, v)\|=\frac{J_{1}}{2} .
\end{aligned}
$$

In a similar manner, we conclude that

$$
\begin{aligned}
T_{2}(u, v)(\mathrm{t}) & =\lambda \int_{0}^{1} H(t, s) \phi_{q}^{-1}\left(\int_{0}^{1} G_{n_{2}}(s, \tau) f_{2}(\tau, u(\tau), v(\tau)) d \tau\right) d s \\
& \geq \frac{1}{2}\|(u, v)\|=\frac{J_{1}}{2} .
\end{aligned}
$$

Therefore, $T(u, v)(t) \geq J_{1}$

Hence $\|T(u, v)\| \geq J_{1}=\|(u, v)\|$ If we set,

$\Omega_{1}=\left\{(\mathrm{u}, \mathrm{v}) \in \mathrm{B} \mid\|(u, v)\|<J_{1}\right\}$,

Then

$\|T(u, v)\| \geq\|(u, v)\|(u, v) \in P \cap \partial \Omega_{1}$

Let $\bar{f}(t) \quad f(t, u(t), v(t)) \bar{f}_{2}(t)=f_{2}(t, u(t), v(t))$. Now, wedefinethe functions $f_{1}^{*}, f_{2}^{*}: \mathrm{R}^{+} \rightarrow \mathrm{R}^{+}$by $f_{1}^{*}(\mathrm{x})=\max _{0 \leq t \leq \phi_{p}(x)} \bar{f}_{1}(t)$ and $f_{2}^{*}(\mathrm{x})=\max _{0 \leq \leq \leq \phi_{q}(x)} \bar{f}_{2}(t)$, for all $\mathrm{x} \in \mathrm{R}^{+}$.

Then $\bar{f}_{1}(t) \leq f_{1}^{*}(\mathrm{x})$ and $\bar{f}_{2}(t) \leq f_{2}^{*}(\mathrm{x})$

It follows that the functions $f_{1}^{*}, f_{2}^{*}$ are non-decreasing and satisfy the conditions

$$
\lim _{x \rightarrow \infty} \frac{f_{1}^{*}(\mathrm{x})}{\phi_{p}(x)} \leq f_{1, \infty} \text { and } \lim _{x \rightarrow \infty} \frac{f_{2}^{*}(\mathrm{x})}{\phi_{q}(x)} \leq f_{2, \infty} .
$$

Next, by the definitions of $f_{1, \infty}$ and $f_{2, \infty}$ there exist $J_{2}>0$ such that

$f_{1}^{*}(\mathrm{x}) \leq\left(f_{1, \infty}+\epsilon\right) \phi_{p}(x)$ and $f_{2}^{*}(\mathrm{x}) \leq\left(f_{2, \infty}+\in\right) \phi_{q}(x), \mathrm{x} \leq \bar{J}_{2}$

Then, for $\mathrm{J}_{2}=\max \left\{2 J_{1}, \bar{J}_{2}\right\}$ we have

$f_{1}^{*}(\mathrm{x}) \leq f_{1}^{*}\left(J_{2}\right)$ and $f_{2}^{*}(\mathrm{x}) \leq f_{2}^{*}\left(J_{2}\right), 0<\mathrm{x} \leq J_{2}$
Choose $(u, v) \in P$ with $\|(u, v)\|=J_{2}$. Then we have

$$
\begin{aligned}
T_{1}(u, v)(\mathrm{t})= & \lambda \int_{0}^{1} H_{m_{1}}(t, s) \phi_{p}^{-1}\left(\int_{0}^{1} G(s, \tau) f_{1}(\tau, u(\tau), v(\tau)) d \tau\right) d s \\
& \leq \lambda \frac{1}{6^{m_{1}-1}} \int_{0}^{1}(1-s) \mathrm{s} \phi_{p}^{-1}\left(\int_{0}^{1} G(\tau, \tau) f_{1}^{*}(x) d \tau\right) d s \\
& \leq \lambda \frac{1}{6^{m_{1}}} \phi_{p}^{-1}\left(\int_{0}^{1} G(\tau, \tau) f_{1}^{*}\left(J_{2}\right) d \tau\right) \\
& \leq \lambda \frac{1}{6^{m_{1}}} \phi_{p}^{-1}\left(\int_{0}^{1} G(\tau, \tau)\left(f_{1, \infty}+\in\right) \phi_{p}\left(J_{2}\right) d \tau\right) \\
& \leq \lambda \frac{1}{6^{m_{1}}} \phi_{p}^{-1}\left(\int_{0}^{1} G(\tau, \tau)\left(f_{1, \infty}+\in\right) \phi_{p}\left(J_{2}\right) d \tau\right) \\
& \leq \lambda \frac{1}{6^{m_{1}}} \phi_{p}^{-1}\left(\int_{0}^{1} G(\tau, \tau)\left(f_{1, \infty}+\in\right) d \tau\right) J_{2} \\
& \leq \frac{J_{2}}{2}
\end{aligned}
$$

In a similar manner, we conclude that

$$
\begin{aligned}
T_{2}(u, v)(\mathrm{t}) & =\lambda \int_{0}^{1} H(t, s) \phi_{q}^{-1}\left(\int_{0}^{1} G_{n_{2}}(s, \tau) f_{2}(\tau, u(\tau), v(\tau)) d \tau\right) d s \\
& \leq \frac{J_{2}}{2} .
\end{aligned}
$$

Therefore, $T(u, v)(t) \geq J_{2}$

Hence, $\|\mathrm{T}(\mathrm{u}, \mathrm{v})\| \leq \mathrm{J} 2=\|(\mathrm{u}, \mathrm{v})\|$. If we set

$\Omega_{2}=\left\{(\mathrm{u}, \mathrm{v}) \in \mathrm{B}\|\|(u, v) \|<J_{2}\right\}$,

And $\|T(u, v)\| \leq\|(u, v)\|(u, v) \in P \cap \partial \Omega_{2}$

Applying Theorem 2.9 to (3.6) and (3.7), we obtain that $T$ has a fixed point $(u, v)$ in $P \cap\left(\overline{\Omega_{2}} \backslash \Omega_{1}\right)$ and hence the BVP (1.1)-(1.4) has a positive solution such that $J_{1} \leq\|(u, v)\| \leq J_{2}$. The proof is complete.

\section{Example}

Let us consider an example to illustrate the above result. Now, we consider two-point (p, q) Laplacian boundary value problems

$$
\begin{aligned}
& (-1)^{1}\left[\phi_{p}\left(u^{(4)}(t)\right)\right]^{\prime \prime \prime}=\lambda f_{1}(t, u(t), v(t)), t \in[0,1], \\
& (-1)^{2}\left[\phi_{q}\left(v^{\prime \prime}(t)\right)\right]^{(6)}=\lambda f_{2}(t, u(t), v(t)), \frac{1}{p}+\frac{1}{q}=1,
\end{aligned}
$$

$$
\left.\begin{array}{c}
u^{(2 i)}(0)=0=u^{(2 i)}(1), \mathrm{i}=0,1, \\
{\left[\phi_{\mathrm{p}}\left(u^{(4)}(\mathrm{t})\right)\right]_{a t t=0}^{(j)}=0, j=0,1,} \\
{\left[\phi_{\mathrm{p}}\left(u^{(4)}(\mathrm{t})\right)\right]_{a t t=1}=0,}
\end{array}\right\}
$$

$$
\left.\begin{array}{c}
{\left[\phi_{q}\left(v^{\prime \prime}(t)\right)\right]_{a t t=0}^{(2 i)}=0=\left[\phi_{q}\left(v^{\prime \prime}(t)\right)\right]_{\text {at } t=1}^{(2 i)}, i=0,1,2,} \\
v(0)=0, v(1)=0
\end{array}\right\}
$$

Where

$$
\begin{aligned}
& f_{1}(t, u(t), v(t))=(u+v)\left[9995000000-9994999845 e^{-5(u+v)^{3}}\right], \\
& f_{2}(t, u(t), v(t))=(u+v)\left[2400000000-2399999970 e^{-7(u+v)^{5}}\right] .
\end{aligned}
$$

The Green's function $G(t, s)$ for the homogeneous BVP, 
Citation: Prasad KR, Kumar KR, Murali P (2014) Solvability of Higher Order (p,q) Laplacian Two-Point Boundary Value Problems. J Appl Computat Math 3: 182. doi:10.4172/2168-9679.1000182

Page 6 of 6

$$
\begin{aligned}
& -x^{\prime \prime \prime}(t)=0, \\
& x(0)=x^{\prime}(0)=0, x(1)=0,
\end{aligned}
$$

is given by

$$
G(t, s)= \begin{cases}\frac{t^{2}(1-s)^{2}}{2}, & 0 \leq t \leq s<1, \\ \frac{t^{2}(1-s)^{2}}{2}-\frac{(t-s)^{2}}{2}, & 0<s \leq t \leq 1 .\end{cases}
$$

The Green's function $H(t, s)$ for the homogeneous BVP,

$$
\begin{aligned}
& -x^{\prime \prime \prime}(t)=0, \\
& x(0)=0, x(1)=0,
\end{aligned}
$$

is given by

$$
H(t, s)= \begin{cases}t(1-s), & 0 \leq t \leq s<1, \\ s(1-t), & 0<s \leq t \leq 1 .\end{cases}
$$

By direct calculations, we have

$$
M=0.0001678466(p=2), \quad \int_{0}^{1} G(\tau, \tau) d \tau=\frac{1}{60}, \quad \int_{0}^{1} H(\mathrm{~s}, \mathrm{~s}) d s=\frac{1}{6},
$$

$f_{1,0}=155, f_{2,0}=90$,

$$
f_{1, \infty}=9995000000, \quad f_{2, \infty}=2400000000,
$$$$
R_{1}=\max \{3.835473314,3.108280305\}=3.835473314 \text {, }
$$

And $R_{2}=\min \{6.967742005,7.2\}=6.967742005$.

Applying Theorem 3.1, we get an optimal eigenvalue interval $3.835473314<\lambda<6.967742005$ for which the boundary value problem (4.1)-(4.4) has at least one positive solution [14-19].

\section{References}

1. Erbe LH, Wang H (1994) On the existence of positive solutions of ordinary differential equations. Proc Amer Math Soc 120: 743-748.
2. Eloe PW, Henderson J (1998) Positive solutions and nonlinear (k, n-k) conjugate eigenvalue problems. Diff Equ Dyna Syst 6: 309-317.

3. Wong PJY, Agarwal RP (1999) Eigenvalues of lidstone boundary value problems. App Math Computation 104: 15-31.

4. Davis JM, Henderson J, Prasad KR, Yin W (2000) Eigenvalue intervals for nonlinear right focal problems. Appl Anal 74: 215-231.

5. Henderson J, Wang $H$ (2007) An eigenvalue problem for quasilinear systems. Rocky Mountain J Math 37.

6. Henderson J, Ntouyas SK, Purnaras IK (2008) Positive solutions for systems of generalized three-point nonlinear boundary value problems. Comment Math Univ Carolin 1: 79-91.

7. Li W (2010) Positive solutions for higher order singular nonlinear ordinary differential systems. Inter J Nonlinear Sci 9 1: 28-40.

8. Agarwal RP, Bohner M, Wong PJY (1999) Positive solutions and eigen values of conjugate boundary value problems, Proc Edinb Math Soc 42: 349-374.

9. Agarwal RP, Lu H, Regan OD (2002) Eigenvalues and the one dimensional p-laplacian. J Math Anal Appl 266: 383-400.

10. Avery R, Henderson J (2004) Existence of three positive pseudo symmetric solutions for a one dimensional p-Laplacian. J Math Anal Appl 277: 395-404.

11. Chu J, Zhou Z (2009) Positive solutions and eigen values of nonlocal boundary value problems. Elect J Diff Equ 86: 1-9.

12. Chu J, O'Regan D, Zhang M (2007) Positive solutions and eigen value intervals for nonlinear systems. Proc Indian Acad Sci 117 1: 85-95.

13. Prasad KR, Murali P, Kumar KR (2013) Existence of positive solutions for higher order ( $p, q)$-Laplacian two-Point boundary value problems. Inter J Diff Equ.

14. Krasnosel'skii MA (1964) Positive Solutions of Operator Equations. P Noordhoff Ltd, Groningen, The Netherlands.

15. Agarwal RP, O'Regan D, Wong PJY (1999) Positive Solutions of Differential, Difference and Integral Equations. Kluwer Academic Publishers, Dordrecht, The Netherlands.

16. Chu J, Jiang D (2005) Eigenvalues and discrete boundary value problems for the one-dimensional p-laplacian. J Math Anal Appl 305: 452-465.

17. Guo D, Lakshmikantam V (1988) Nonlinear Problems in Abstract Cones Academic press, San Diego.

18. Infante G (2003) Eigen values of some non-local boundary value problems Proc Edinb Math Soc 46: 75-86.

19. Wang $H$ (2003) On the number of positive solutions of nonlinear systems. J Math Anal Appl 281: 287-306. 